\title{
Revisiting the role of dose escalation in esophageal cancer in the era of modern radiation delivery
}

\author{
Chukwuka Eze ${ }^{1}$, Nina-Sophie Schmidt-Hegemann ${ }^{1}$, Lino Morris Sawicki ${ }^{2}$, Franziska Walter ${ }^{1}$, \\ Farkhad Manapov ${ }^{1,3}$
}

${ }^{1}$ Department of Radiation Oncology, University Hospital, LMU Munich, Munich, Germany; ${ }^{2}$ Department of Diagnostic and Interventional Radiology, Medical Faculty, University Dusseldorf, Dusseldorf, Germany; ${ }^{3}$ Comprehensive Pneumology Center Munich (CPC-M), Member of the German Center for Lung Research (DZL), Munich, Germany

Correspondence to: Chukwuka Eze, MD. Department of Radiation Oncology, University Hospital, LMU Munich, Marchioninistrasse 15, 81377 Munich, Germany. Email: Chukwuka.Eze@med.uni-muenchen.de.

Provenance and Peer Review: This article was commissioned and reviewed by the Section Editor Xiaozheng Kang (Department of Thoracic Surgery, Beijing Cancer Hospital, Peking University, Beijing, China).

Comment on: Chen D, Menon H, Verma V, et al. Results of a Phase 1/2 Trial of Chemoradiotherapy With Simultaneous Integrated Boost of Radiotherapy Dose in Unresectable Locally Advanced Esophageal Cancer. JAMA Oncol 2019. [Epub ahead of print].

Submitted Dec 29, 2019. Accepted for publication Feb 02, 2020.

doi: $10.21037 /$ jtd.2020.02.38

View this article at: http://dx.doi.org/10.21037/jtd.2020.02.38

Historically, the landmark 1992 RTOG 85-01 trial by Herskovic et al. established concurrent chemoradiation (CRT) to a total dose of $50 \mathrm{~Gy}$ with cisplatin and fluorouracil as superior to 64 Gy radiation therapy alone (1). Additional studies have also demonstrated durable local control rates after delivery of $>50.4$ Gy CRT with/without brachytherapy boost $(2,3)$. The seminal INT 0123 trial (RTOG 94-05) published in 2002 was a phase III trial comparing high-dose (64.8 Gy) vs. standard dose (50.4 Gy) CRT with concurrent 5 -fluorouracil and cisplatin chemotherapy in cT1-T4, cN0/1, M0 esophageal cancer selected for a nonsurgical approach (4). The trial was terminated early after an interim analysis due to futility. The median follow-up was 16.4 months for all patients and 29.5 months for patients still alive. Of the 218 randomized patients [squamous cell carcinoma (SCC): $85 \%$, adenocarcinoma (ADC): 15\%], median survival, 2-year OS and 2-year locoregional disease persistence/failure were 13.0 vs. 18.1 months, $31 \%$ vs. $40 \%$ and $56 \%$ vs. $52 \%$ in the high-dose and standard-dose arms, respectively (nonsignificant in all instances). Seven of the 11 deaths in the high-dose arm occurred in patients who received $\leq 50.4$ Gy. Thus, the trial has been subject to critique as some authors have commented that the high mortality in the highdose arm may not be related to the applied radiation dose, given that most of these deaths occurred in patients who had not reached the dose escalation portion of treatment and this may have obscured the results. In addition, critics have argued that older radiation techniques were utilized, and that dose escalation should be evaluated using modern radiation techniques. The results of the aforementioned trial were however corroborated by a recent analysis of the National Cancer Data Base (NCDB) by Brower et al. (5). From 2004 to 2012, a total of 6,854 patients were included; 3,821 (55.7\%) received 50-50.4 Gy and 3,033 (44.3\%) received doses $>50.4$ Gy. There was no significant difference in OS in patients receiving 50 to 50.4 Gy $v$ s. $>50.4$ Gy $(\mathrm{P}=0.53)$. On subgroup analyses, the effect of dose escalation based on histology and delivery of intensity modulated radiation therapy (IMRT) demonstrated no benefit, which was also confirmed on propensity score matching analysis. Interestingly, the data highlighted that many radiation oncologists had not universally embraced the concept that dose escalation was not associated with improved OS in esophageal cancer given the number of patients receiving >50.4 Gy. Thus, radiation dose escalation in esophageal cancer has long been understudied, although modest improvements in survival have been achieved by combining neoadjuvant CRT and surgery (6). However, survival in patients treated with CRT or surgery alone 
remains dismal with unacceptably high locoregional recurrence and mortality rates. As such, the issue of doseescalation has been revisited.

The phase I/II trial of CRT with simultaneous integrated boost (SIB) in unresectable advanced esophageal cancer is one of such studies (7). In the study by Chen et al., 46 patients with locally advanced or metastatic esophageal cancer were enrolled from April 2010 to April 2015 at a single tertiary academic cancer center to receive CRT to a total dose of 50.4/63.0 Gy in 28 daily fractions to subclinical regions at risk and SIB to the gross tumor and involved nodes. After an initial total SIB dose of 58.8 Gy (evaluation of toxicity in the first 3 patients), 63.0 Gy was delivered in the rest of the patient cohort if no grade 4-5 adverse events were observed. As part of an interim analysis, a comparison with 97 similar patients treated from January 2010 to December 2014 at the same institution was performed. At a median follow-up of 52 months, the 6-month, 1-year and 2 -year local recurrence rates were $22 \%, 30 \%$, and $33 \%$, respectively. Median overall survival time was 21.5 months. Again, exploratory comparison with the 97-patient cohort receiving standard-dose CRT demonstrated improved local control [hazard ratio (HR), 0.49; 95\% CI, 0.26-0.92; $\mathrm{P}=0.03]$ and $\mathrm{OS}$ (HR, 0.66; 95\% CI, 0.47-0.94; $\mathrm{P}=0.02$ ) in the group that received CRT with a SIB. However, based on the pre-determined primary endpoint, enrolment of at least 42 patients would provide $90 \%$ power to detect an improvement in 1-year tumor control from $70 \%$ to $85 \%$ ( 1 -sided $\alpha<0.10)$. The authors concluded that although the results of this trial did not meet the pre-specified target, it should not be considered negative. The reasoning being that if the reference 1-year LC had been approximately $55 \%$ observed in the INT- 0123 trial, the results of the current trial would be deemed superior.

Nevertheless, corroborative prospective randomized controlled multicenter trials are required to test this modern treatment paradigm. Several studies have been initiated and will be addressed later in this editorial commentary.

This analysis by Chen et al. is interesting and highly relevant as the authors address an issue which has long puzzled radiation oncologists. The authors should be commended for conducting this study. The results of 2-year locoregional control of $67 \%$ are encouraging. Importantly, treatment was well tolerated with 10 acute grade 3 adverse events constituting dysphagia $(n=3)$, anorexia $(n=3)$ and esophagitis $(n=4)$ as well as 3 late grade 3 toxic events. No patients experienced grade $4 / 5$ adverse events. Vis-à-vis all long-term events (regardless of grade), 17 patients (37\%) developed esophageal stricture. There was no difference when compared to the rate of stricture development in the 97-patient contemporarily treated cohort with standard dose [30 (31\%); $\mathrm{P}=0.47$ ]. Two patients developed selflimited (grade 1 or 2 ) esophageal hemorrhage. This may yet have been underestimated due to inclusion of patients who eventually received surgery, as both local failure (relative to definitive CRT) and areas at risk of late toxic effects (e.g., stricture) are reduced by esophagectomy. Nevertheless, one may conclude that dose-escalation via SIB to gross tumor volume and involved lymph nodes up to 63 Gy in 28 daily fractions appears to be safe and effective.

Initial staging included contrast-enhanced positron emission tomography-computed tomography (PET-CT) for all enrolled patients. PET-CT in esophageal cancer has the ability to better detect distant metastases not fully appreciated on CT imaging alone (8). PET-CT has also been utilized for tumor volume delineation (TVD) in esophageal cancer (9). Furthermore, the study was performed at a high-volume tertiary cancer center and inclusion of a high proportion of patients with advanced disease (87\% cT3T4, 78\% stage III-IV) is a major bonus. Concurrent CRT was also delivered in all patients with the use of modern radiation treatment techniques: IMRT (photon) in $39(85 \%)$ patients and intensity-modulated proton therapy (IMPT) in $7(15 \%)$ patients. All patients underwent daily image guidance prior to each radiation fraction. In addition, the median follow-up of 52 months is another strength of the analysis as well as the additional exploratory comparison of treatment efficacy in a 97 contemporarily treated cohort at the same institution, which in fairness is no substitute for a prospective randomized trial but provides some valuable insights.

Notwithstanding, the current analysis however has some limitations in addition to those noted by the authors. The study was a mono-centric study enrolling a limited number of patients (in total 46 patients) with $8(17 \%)$ patients presenting with oligometastatic disease, defined as cases with up to 3 distant metastatic sites; all had nonregional nodal disease but no visceral organ metastasis. Also, multimodal treatment was heterogeneous with 17 (37\%) patients receiving induction chemotherapy prior to concurrent CRT and 11 (24\%) patients eventually undergoing esophagectomy. Other caveats of the present include missing information regarding anatomical localization of the primary tumor: cervical $v s$. thoracic. There is no supplementary information on radiation treatment and quality assurance e.g., mean heart and lung 
doses as well as the volume of heart or lung receiving 20 and 30 Gy, respectively (V20 and V30) although the authors report that no patient experienced late (regardless of grade) cardiopulmonary adverse events and no patient experienced intraoperative complications, although postoperative cardiopulmonary complications included atrial fibrillation, acute respiratory distress syndrome and aspiration pneumonia. None of the 11 patients that underwent surgery died within the first 90 days after surgery. In surgical patients, previous studies addressing preoperative CRT followed by resection showed that dosimetric parameters including mean lung dose (MLD), effective dose, V5, V10 and V15 were predictors of development of postoperative pulmonary complications $(10,11)$. There is also no data on the rates of severe ( $\geq$ grade 3 ) hematological toxicity including neutropenic fever etc. as well as patient treatment compliance. Previous data have revealed that the addition of chemotherapy can also significantly increase acute complications. In a follow-up analysis of the RTOG 8501 study, patients in the concurrent CRT arm had a higher incidence of acute grade 3 (44\% vs. $25 \%)$ and grade 4 toxicity (20\% vs. 3\%) (12).

Importantly, in an era in which biomarkers of treatment response are being intensively investigated, information on such analyses is not provided. Previously, the same group at MD Anderson Cancer Center (MDACC) showed that grade 4 lymphopenia during CRT and not lymphocyte recovery post-CRT independently predicted worse OS in esophageal cancer patients (13).

The search for biomarkers predicting treatment efficacy should be deemed a pre-requisite for further optimization of multimodal treatment, especially molecular-genetic and immunological factors characterizing tumor and patient constitution may play a role in long-term tumor control and outcome after chemoradiation. Future studies will need to address these critical points.

Currently, several ongoing phase III studies are evaluating the concept of dose-escalation. The French CONCORDE-PRODIGE 26 trial (NCT01348217) is currently randomizing patients to receive 66 vs. $50 \mathrm{~Gy}$ combined with FOLFOX $4(14,15)$. Similarly, the Dutch ART deco trial (old NTR ID: 3532) is evaluating radiation dose escalation in non-operable esophageal cancer patients (50.4 vs. 61 Gy with increasing dose per fraction concomitant boost) with concurrent carboplatin and paclitaxel. The United Kingdom SCOPE 2 (NCT02741856) study is a four-arm trial comparing carboplatin/paclitaxel versus cisplatin-/capecitabine-based
CRT, with a second randomization to standard $v$ s. highdose radiation therapy (50 vs. $60 \mathrm{~Gy}$, both in 25 fractions). Patients will receive induction chemotherapy and then be further randomized based on interim PET analyses.

Finally, the role of proton beam therapy (PBT) in the multimodal treatment of esophageal cancer has previously been addressed in a retrospective study at the MDACC. Compared with IMRT, PBT was associated with improved OS, PFS and locoregional failure-free survival (LRFFS) (16). A number of prospective randomized studies are ongoing and comparing PBT vs. IMRT (NCT03801876, NCT01512589). The study by Chen et al. although in a limited number of patients did not report a survival advantage of IMPT vs. IMRT $(\mathrm{P}=0.22)$, however, local recurrence cumulative incidence at 12 months was $14 \%$ and $33 \%$ in the IMPT and IMRT groups, respectively.

In conclusion, in our opinion the issue of dose escalation in esophageal cancer addressed in the study by Chen $e t$ al. has long been a conundrum for radiation oncologists. Based on their findings, dose-escalation seems feasible, but the results of prospective randomized studies will have to be awaited before it can be considered standard of care.

\section{Acknowledgments}

Funding: None.

\section{Footnote}

Conflicts of Interest: All authors have completed the ICMJE uniform disclosure form (available at http://dx.doi. org/10.21037/jtd.2020.02.38). The authors have no conflicts of interest to declare.

Ethical Statement: The authors are accountable for all aspects of the work in ensuring that questions related to the accuracy or integrity of any part of the work are appropriately investigated and resolved.

Open Access Statement: This is an Open Access article distributed in accordance with the Creative Commons Attribution-NonCommercial-NoDerivs 4.0 International License (CC BY-NC-ND 4.0), which permits the noncommercial replication and distribution of the article with the strict proviso that no changes or edits are made and the original work is properly cited (including links to both the formal publication through the relevant DOI and the license). See: https://creativecommons.org/licenses/by-nc-nd/4.0/. 


\section{References}

1. Herskovic A, Martz K, Al-Sarraf M, et al. Combined Chemotherapy and Radiotherapy Compared with Radiotherapy Alone in Patients with Cancer of the Esophagus. N Engl J Med 1992;326:1593-8.

2. Murakami M, Kuroda Y, Okamoto Y, et al. Neoadjuvant concurrent chemoradiotherapy followed by definitive high- dose radiotherapy or surgery for operable thoracic esophageal carcinoma. Int J Radiat Oncol Biol Phys 1998;40:1049-59.

3. Burmeister BH, Dickie G, Smithers BM, et al. Thirty-four patients with carcinoma of the cervical esophagus treated with chemoradiation therapy. Arch Otolaryngol Head Neck Surg 2000;126:205-8.

4. Minsky BD, Pajak TF, Ginsberg RJ, et al. INT 0123 (Radiation Therapy Oncology Group 94-05) Phase III Trial of Combined-Modality Therapy for Esophageal Cancer: High-Dose Versus Standard-Dose Radiation Therapy. J Clin Oncol 2002;20:1167-74.

5. Brower JV, Chen S, Bassetti MF, et al. Radiation Dose Escalation in Esophageal Cancer Revisited: A Contemporary Analysis of the National Cancer Data Base, 2004 to 2012. Int J Radiat Oncol Biol Phys 2016;96:985-93.

6. van Hagen P, Hulshof MCCM, van Lanschot JJB, et al. Preoperative Chemoradiotherapy for Esophageal or Junctional Cancer. N Engl J Med 2012;366:2074-84.

7. Chen D, Menon H, Verma V, et al. Results of a Phase $1 / 2$ Trial of Chemoradiotherapy with Simultaneous Integrated Boost of Radiotherapy Dose in Unresectable Locally Advanced Esophageal Cancer. JAMA Oncol 2019. [Epub ahead of print].

8. Van Westreenen HL, Westerterp M, Bossuyt PMM, et al. Systematic review of the staging performance of $18 \mathrm{~F}$ fluorodeoxyglucose positron emission tomography in esophageal cancer. J Clin Oncol 2004;22:3805-12.

9. Wu AJ, Bosch WR, Chang DT, et al. Expert consensus

Cite this article as: Eze C, Schmidt-Hegemann NS, Sawicki LM, Walter F, Manapov F. Revisiting the role of dose escalation in esophageal cancer in the era of modern radiation delivery. J Thorac Dis 2020;12(4):1624-1627. doi: 10.21037/jtd.2020.02.38 contouring guidelines for intensity modulated radiation therapy in esophageal and gastroesophageal junction cancer. Int J Radiat Oncol Biol Phys 2015;92:911-20.

10. Lee HK, Vaporciyan AA, Cox JD, et al. Postoperative pulmonary complications after preoperative chemoradiation for esophageal carcinoma: Correlation with pulmonary dose-volume histogram parameters. Int J Radiat Oncol Biol Phys 2003;57:1317-22.

11. Tucker SL, Liu HH, Wang S, et al. Dose-volume modeling of the risk of postoperative pulmonary complications among esophageal cancer patients treated with concurrent chemoradiotherapy followed by surgery. Int J Radiat Oncol Biol Phys 2006;66:754-61.

12. al-Sarraf M, Martz K, Herskovic A, et al. Progress report of combined chemoradiotherapy versus radiotherapy alone in patients with esophageal cancer: An intergroup study. J Clin Oncol 1997;15:277-84.

13. Deng $\mathrm{W}, \mathrm{Xu} \mathrm{C}$, Liu A, et al. The relationship of lymphocyte recovery and prognosis of esophageal cancer patients with severe radiation-induced lymphopenia after chemoradiation therapy. Radiother Oncol 2019;133:9-15.

14. Crehange G, Bonnetain F, Peiffert D, et al. Phase II/III randomized trial of exclusive chemoradiotherapy with or without dose escalation in locally advanced esophageal carcinoma: The CONCORDE study (PRODIGE 26). J Clin Oncol 2016;34:TPS190.

15. Crehange G, Bertaut A, Peiffert D, et al. Exclusive chemoradiotherapy with or without dose escalation in locally advanced esophageal carcinoma: The CONCORDE study (PRODIGE 26). J Clin Oncol 2017;35:4037.

16. Xi M, Xu C, Liao Z, et al. Comparative Outcomes After Definitive Chemoradiotherapy Using Proton Beam Therapy Versus Intensity Modulated Radiation Therapy for Esophageal Cancer: A Retrospective, SingleInstitutional Analysis. Int J Radiat Oncol Biol Phys 2017;99:667-76. 